REPRESENTATION THEORY

An Electronic Journal of the American Mathematical Society

Volume 15, Pages 244-257 (March 14, 2011)

S $1088-4165(2011) 00385-X$

\title{
FINITE GENERATION OF TATE COHOMOLOGY
}

\author{
JON F. CARLSON, SUNIL K. CHEBOLU, AND JÁN MINÁČ
}

Dedicated to Professor Luchezar Avramov on his sixtieth birthday.

\begin{abstract}
Let $G$ be a finite group and let $k$ be a field of characteristic $p$. Given a finitely generated indecomposable nonprojective $k G$-module $M$, we conjecture that if the Tate cohomology $\hat{H}^{*}(G, M)$ of $G$ with coefficients in $M$ is finitely generated over the Tate cohomology ring $\hat{H}^{*}(G, k)$, then the support variety $V_{G}(M)$ of $M$ is equal to the entire maximal ideal spectrum $V_{G}(k)$. We prove various results which support this conjecture. The converse of this conjecture is established for modules in the connected component of $k$ in the stable Auslander-Reiten quiver for $k G$, but it is shown to be false in general. It is also shown that all finitely generated $k G$-modules over a group $G$ have finitely generated Tate cohomology if and only if $G$ has periodic cohomology.
\end{abstract}

\section{INTRODUCTION}

Tate cohomology was introduced by Tate in his celebrated paper 14 where he proved the main theorem of class field theory in a remarkably simple way using Tate cohomology. After Cartan and Eilenberg's treatment 9] of Tate cohomology and Swan's basic results on free group actions on spheres [13, Tate cohomology became one of the basic tools in current mathematics. Our aim in this paper is to address a fundamental question: When is the Tate cohomology with coefficients in a module finitely generated over the Tate cohomology ring of the group?

Suppose $G$ be a finite group and let $k$ be a field of characteristic $p$. If $M$ is a finitely generated $k G$-module, then a well-known result in group cohomology due to Golod, Evens and Venkov says that $\mathrm{H}^{*}(G, M)$ is finitely generated as a graded module over $\mathrm{H}^{*}(G, k)$. Our goal is to investigate a similar finite-generation result for Tate cohomology. More precisely, if $M$ is a finitely generated $k G$-module, then we want to know whether the Tate cohomology $\hat{\mathrm{H}}^{*}(G, M)$ of $G$ with coefficients in $M$ is finitely generated as a graded module over the Tate cohomology $\operatorname{ring} \hat{\mathrm{H}}^{*}(G, k)$. In Section 2 we explain one reason for being interested in this problem. In general, it seems that the Tate cohomology of a module is seldom finitely generated, which is a striking contrast to the situation with ordinary cohomology. However, there are some notable exceptions. Our investigations have led us to a conjecture which we state as follows.

Received by the editors August 17, 2009 and, in revised form, March 9, 2010.

2010 Mathematics Subject Classification. Primary 20C20, 20J06; Secondary 55P42.

Key words and phrases. Tate cohomology, finite generation, periodic modules, support varieties, stable module category, almost split sequence.

The first author is partially supported by a grant from NSF and the third author is supported from NSERC.

(C)2011 American Mathematical Society Reverts to public domain 28 years from publication 
Conjecture 1.1. Let $G$ be a finite group and let $M$ be an indecomposable finitely generated $k G$-module such that $\mathrm{H}^{*}(G, M) \neq\{0\}$. If $\hat{\mathrm{H}}^{*}(G, M)$ is finitely generated over $\hat{\mathrm{H}}^{*}(G, k)$, then the support variety $V_{G}(M)$ of $M$ is equal to the entire maximal ideal spectrum $V_{G}(k)$ of the group cohomology ring.

The condition that $\mathrm{H}^{*}(G, M) \neq 0$ is certainly necessary since there are many modules with proper support varieties and vanishing cohomology [5]. Perhaps it is necessary to require that $M$ lies in the thick subcategory of the stable category generated by $k$.

We have evidence for the conjecture from two directions. First, the results of [3] indicate that products in negative Tate cohomology are often zero and we can use this to develop boundedness conditions on finitely generated modules over Tate cohomology. Under the right circumstances, these conditions imply infinite generation of the Tate cohomology. Second, for groups having $p$-rank at least two, we can show that many periodic modules fail to have finitely generated Tate cohomology. Indeed, we prove that for any such group there is at least one module whose Tate cohomology is not finitely generated. Hence, the only groups having the property that every finitely generated $k G$-module has finitely generated Tate cohomology have $p$-rank one or zero.

On the other hand, in general, there are numerous modules which have finitely generated Tate cohomology. In the last section we show some ways in which these modules can be constructed. It turns out that the constructions are consistent with the Auslander-Reiten quiver for $k G$-modules. That is, if a nonprojective module in a connected component of the Auslander-Reiten quiver has finitely generated Tate cohomology, then so does every module in that component.

The paper is organized as follows. We begin in Section 2 by explaining how we had naturally arrived at the problem of finite generation of Tate cohomology. Sections 3 and 4 deal with modules whose Tate cohomology is not finitely generated and contain proofs in the two directions mentioned above. In Section 5 we prove affirmative results which provides a good source of modules whose Tate cohomology is finitely generated.

Throughout the paper $G$ denotes a nontrivial finite group, and all $k G$-modules are assumed to be finitely generated. We use standard facts and notation of the stable module category of $k G[7$, support varieties [2, 8], and of almost split sequences [2].

\section{Universal GHOSts IN $\operatorname{stmod}(k G)$}

Here we explain briefly how we had arrived at the problem of finite generation of Tate cohomology. More details can be found in 10, 11. The question of finite generation is very natural. The finite generation of the ordinary cohomology has been very important in the development of the theory of support varieties and in other connections. For Tate cohomology, almost nothing is known about the question of finite generation beyond what is in this paper.

The following natural question was raised in [11: When does the Tate cohomology functor detect trivial maps in the stable module category $\operatorname{stmod}(k G)$ of finitely generated $k G$-modules? A map $\phi: M \rightarrow N$ between finitely generated $k G$-modules is said to be a ghost if the induced map in Tate cohomology groups

$$
\underline{\operatorname{Hom}}_{k G}\left(\Omega^{i} k, M\right) \longrightarrow \underline{\operatorname{Hom}}_{k G}\left(\Omega^{i} k, N\right)
$$


is zero for each integer $i$. With this definition, the above question is equivalent to asking: When is every ghost map in $\operatorname{stmod}(k G)$ trivial? In addressing this question, it is convenient to have a universal ghost out of any finitely generated $k G$-module $M$ in $\operatorname{stmod}(k G)$, i.e., a ghost map $\phi: M \rightarrow N$ in $\operatorname{stmod}(k G)$ such that every ghost out of $M$ factors through $N$ via $\phi$. The point is that if the universal ghost vanishes, then all ghosts vanish.

So the problem boils down to finding a universal ghost out of $M$ (if it exists) for every module $M$ in $\operatorname{stmod}(k G)$. The point is that if the Tate cohomology $\hat{\mathrm{H}}^{*}(G, M)$ is finitely generated as a graded module over $\hat{\mathrm{H}}^{*}(G, k)$, then a universal ghost out of $M$ can be constructed in $\operatorname{stmod}(k G)$. This is done as follows. Let $\left\{v_{j}\right\}$ be a finite set of homogeneous generators for $\hat{\mathrm{H}}^{*}(G, M)$ as a $\hat{\mathrm{H}}^{*}(G, k)$-module. These generators can be assembled into a map

$$
\bigoplus_{j} \Omega^{\left|v_{j}\right|} k \longrightarrow M
$$

in $\operatorname{stmod}(k G)$. This map can then be completed to a triangle

$$
\bigoplus_{j} \Omega^{\left|v_{j}\right|} k \longrightarrow M \stackrel{\Psi_{M}}{\longrightarrow} F_{M}
$$

By construction, it is clear that the first map in the above triangle is surjective on the functors $\underline{\operatorname{Hom}}_{k G}\left(\Omega^{l} k,-\right)$ for each $l$. Therefore, the second map $\Psi_{M}$ must be a ghost. Thus we have the following proposition.

Proposition 2.1. Suppose that $M$ is a finitely generated $k G$-module such that $\hat{\mathrm{H}}^{*}(G, M)$ is finitely generated as a graded module over $\hat{\mathrm{H}}^{*}(G, k)$. Then the map $\Psi_{M}: M \rightarrow F_{M}$ in the above triangle is a universal ghost out of $M$.

Proof. Universality of $\Psi_{M}$ is easy to see. For the last statement, we note that because the sum is finite, $\bigoplus_{j} \Omega^{\left|v_{j}\right|} k$ is finitely generated.

\section{Modules With BOUNdS ON Finitely GENERATED SUBMOdulES}

In this section we apply our main method for showing that modules over Tate cohomology are not finitely generated. We explore the implications of the following condition.

The material in this section draws heavily on the methods introduced in the paper 3 .

Definition 3.1. We say that a graded module $T=\bigoplus_{n \in \mathbb{Z}} T^{n}$ over $\hat{\mathrm{H}}^{*}(G, k)$ has bounded finitely generated submodules if for any $m$ there is a number $N=N(m)$ such that the submodule $S$ of $T$ generated by $\bigoplus_{n>m} T^{n}$ is contained in $\bigoplus_{n>N} T^{n}$.

Lemma 3.2. If a graded module $T=\bigoplus_{n \in \mathbb{Z}} T^{n}$ over $\hat{\mathrm{H}}^{*}(G, k)$ has bounded finitely generated submodules and if $T^{n} \neq\{0\}$ for arbitrarily small (meaning negative) values of $n$, then $T$ is not a finitely generated module over $\hat{\mathrm{H}}^{*}(G, k)$.

Proof. The proof is an immediate consequence of the definition. The point is that any finitely generated submodule of $T$ is contained in $\sum_{n>m} T^{n}$ for some $m$ and hence cannot generate all of $T$. 
Remark 3.3. There is a more general formulation of the boundedness condition that might be useful, though we do not use it in this paper. We say that $T^{*}$ has submaximal growth of finitely generated submodules if the degree of the pole at 1 of the Poincaré series for the submodule $S$ of $T$ generated by $\bigoplus_{n>m} T^{n}$ is strictly smaller than the degree of the pole at 1 of the Poincaré series of $T$. The Poincaré series for $T$ is the Laurent series

$$
f_{T}(t)=\sum_{n=-\infty}^{\infty}\left(\operatorname{Dim}\left(T^{n}\right)\right) t^{n} .
$$

Its pole at $t=1$ is a measure of the growth rate of $T$ in negative degrees. That is, if the pole has degree $d$, then there is a number $c$ such that $\operatorname{Dim}\left(T^{-n}\right) \leq c n^{d-1}$ for all $n$, while for any constant $c$ there exists a natural number $n$ such that $\operatorname{Dim}\left(T^{-n}\right)>$ $c n^{d-2}$. It is straightforward to show that any $T^{*}$ which has submaximal growth of finitely generated submodule is not finitely generated over $\hat{\mathrm{H}}^{*}(G, k)$.

The graded modules over the Tate cohomology ring that we are interested in have the form $\hat{\mathrm{H}}^{*}(G, L)$, where $L$ is a $k G$-module. We remind the reader that if $\hat{\mathrm{H}}^{i}(G, L) \neq 0$ for some $i$, then it is also nonzero for infinitely many negative and infinitely many positive values of $i$ [5, Thm. 1.1]. Moreover, a standard argument shows that any nonprojective module $L$ in the thick subcategory generated by $k$ has nonvanishing Tate cohomology. Here, the thick subcategory generated by $k$ is the smallest full subcategory of $\operatorname{stmod}(k G)$ that contains $k$ and is closed under exact triangles and direct summands.

We use the next lemma several times in what follows.

Lemma 3.4. Suppose that we have an exact sequence

$$
\mathcal{E}: \quad 0 \longrightarrow L \longrightarrow N \longrightarrow N
$$

of $k G$-modules where $\mathcal{E}$ represents an element $\zeta$ in $\operatorname{Ext}_{k G}^{1}(N, L)$. Cup product with the element $\zeta$ induces a homomorphism $\zeta: \hat{\mathrm{H}}^{*}(G, N) \longrightarrow \hat{\mathrm{H}}^{*}(G, L)[1]$. Let $\mathcal{K}^{*}$ be the kernel of the multiplication by $\zeta$, and let $\mathcal{J}^{*}$ be the cokernel of multiplication by $\zeta$. Then we have an exact sequence of $\hat{\mathrm{H}}^{*}(G, k)$-modules

$$
0 \longrightarrow \mathcal{J}^{*} \longrightarrow \hat{\mathrm{H}}^{*}(G, M) \longrightarrow \mathcal{K}^{*} \longrightarrow 0 .
$$

Moreover, if $\mathcal{K}^{*}$ is not finitely generated over $\hat{\mathrm{H}}^{*}(G, k)$, then neither is $\hat{\mathrm{H}}^{*}(G, M)$.

Proof. The proof is a straightforward consequence of the naturality of the long exact sequence on Tate cohomology. That is, we have a sequence

$$
\ldots \stackrel{\zeta}{\longrightarrow} \hat{\mathrm{H}}^{n}(G, L) \longrightarrow \hat{\mathrm{H}}^{n}(G, M) \longrightarrow \hat{\mathrm{H}}^{n}(G, N) \stackrel{\zeta}{\longrightarrow} \hat{\mathrm{H}}^{n+1}(G, L) \longrightarrow \ldots
$$

and we note that the collection of the maps $\zeta$ in the long exact sequence is a map of degree 1 of $\hat{\mathrm{H}}^{*}(G, k)$-modules

$$
\zeta: \quad \hat{\mathrm{H}}^{*}(G, N) \longrightarrow \hat{\mathrm{H}}^{*}(G, L)[1] .
$$

(The symbol $\mathcal{X}[i]$ indicates the shift of the $\hat{\mathrm{H}}^{*}(G, k)$-module $\mathcal{X}$ by $i$ degrees.)

The last statement is a consequence of the fact that quotient modules of finitely generated modules are finitely generated. 
Now suppose that $\zeta \in \mathrm{H}^{d}(G, k)$ for $d>0$ and that $\zeta \neq 0$. We have an exact sequence

$$
0 \longrightarrow L_{\zeta} \longrightarrow \Omega^{d} k \stackrel{\zeta}{\longrightarrow} k \longrightarrow 0
$$

where $\zeta$ in the sequence is a homomorphism (uniquely) representing the cohomology element $\zeta$. In the corresponding long exact sequence on Tate cohomology

$\ldots \stackrel{\zeta}{\longrightarrow} \hat{\mathrm{H}}^{n-1}(G, k) \longrightarrow \hat{\mathrm{H}}^{n}\left(G, L_{\zeta}\right) \longrightarrow \hat{\mathrm{H}}^{n}\left(G, \Omega^{d} k\right) \stackrel{\zeta}{\longrightarrow} \hat{\mathrm{H}}^{n}(G, k) \longrightarrow \ldots$,

the homomorphism labeled $\zeta$ is multiplication by $\zeta$. That is, it is a degree $d$ map:

$$
\zeta: \hat{\mathrm{H}}^{*}(G, k)[-d] \quad \longrightarrow \quad \hat{\mathrm{H}}^{*}(G, k) \text {. }
$$

Here we are using the fact that $\hat{\mathrm{H}}^{s}\left(G, \Omega^{d} k\right) \cong \hat{\mathrm{H}}^{s-d}(G, k)$.

As a result, we have, as in Lemma 3.4, an exact sequence of $\hat{\mathrm{H}}^{*}(G, k)$-modules,

$$
0 \longrightarrow \mathcal{J}^{*}[-1] \longrightarrow \hat{\mathrm{H}}^{*}\left(G, L_{\zeta}\right) \longrightarrow \mathcal{K}^{*}[-d] \longrightarrow 0,
$$

where $\mathcal{J}^{*}$ and $\mathcal{K}^{*}$ are the cokernel and kernel of multiplication by $\zeta$, respectively.

Lemma 3.5. Suppose that $\zeta \in \mathrm{H}^{d}(G, k)$ is a regular element on $\mathrm{H}^{*}(G, k)$. Then

(1) $\mathcal{K}^{m}=\{0\}$ for all $m \geq 0$, and

(2) $\mathcal{J}^{m}=\{0\}$ for all $m<0$.

Proof. The first statement is the definition that $\zeta$ is a regular element in $\mathrm{H}^{*}(G, k)$. The second statement is a consequence of Lemma 3.5 of [3]. For the sake of completeness we include a proof. For $t>0$, let

$$
\langle,\rangle: \hat{\mathrm{H}}^{-t-1}(G, k) \otimes \hat{\mathrm{H}}^{t}(G, k) \longrightarrow \hat{\mathrm{H}}^{-1}(G, k) \cong k
$$

be the Tate duality. Let $\zeta_{1}, \ldots, \zeta_{s}$ be a $k$-basis for $\hat{\mathrm{H}}^{-m-1}(G, k)$. Then because multiplication by $\zeta$,

$$
\hat{\mathrm{H}}^{-m-1}(G, k) \longrightarrow \hat{\mathrm{H}}^{-m+d-1}(G, k)
$$

is a monomorphism (since $-m-1 \geq 0$ ), the elements $\zeta \zeta_{1}, \ldots, \zeta \zeta_{s}$ are linearly independent. So there must exist elements $\gamma_{1}, \ldots, \gamma_{s}$ in $\hat{\mathrm{H}}^{m-d}(G, k)$ such that for all $i$ and $j$, we have

$$
\left\langle\gamma_{i}, \zeta \zeta_{j}\right\rangle=\left\langle\gamma_{i} \zeta, \zeta_{j}\right\rangle=\delta_{i, j}
$$

where by $\delta_{i, j}$ we mean the usual Kronecker delta. A consequence of this is that the elements $\gamma_{1} \zeta, \ldots, \gamma_{s} \zeta$ must be linearly independent and hence must form a basis for $\hat{\mathrm{H}}^{m}(G, k)$. This proves the lemma.

There are many examples of groups for which all products in negative cohomology are zero. For example, we remind the reader of the following theorem from [3].

Theorem 3.6. Suppose that the ordinary cohomology ring $\mathrm{H}^{*}(G, k)$ has a regular sequence of length 2. Then the product of any two elements in negative cohomology is zero. In particular, this happens whenever the p-rank of the center of a Sylow p-subgroup of $G$ is at least 2.

The second statement of the theorem was proved by Duflot (see Theorem 12.3.3 of [8]). 
Proposition 3.7. Suppose that $G$ has p-rank at least two and that $\hat{\mathrm{H}}^{*}(G, k)$ has the property that the product of any two elements in negative degrees is zero. If $\zeta \in \mathrm{H}^{d}(G, k)(d>0)$ is a regular element for $\mathrm{H}^{*}(G, k)$, then $\hat{\mathrm{H}}^{*}\left(G, L_{\zeta}\right)$ is not finitely generated as a module over $\hat{\mathrm{H}}^{*}(G, k)$.

Proof. As before, let $\mathcal{K}^{*}$ be the kernel of the multiplication by $\zeta$ on $\hat{\mathrm{H}}^{*}(G, k)$. The fact that $\mathcal{K}^{*}$ is not zero in infinitely many negative degrees follows easily from Lemma 2.1 of [3] and the fact that there is no bound on the dimensions of the spaces $\hat{\mathrm{H}}^{n}(G, k)$ for negative values of $n$. We have shown that $\mathcal{K}^{*}$ has elements only in negative degrees and products of elements in negative degrees are zero. Therefore, $\mathcal{K}$ has bounded finitely generated submodules and by Lemma 3.2 it is not finitely generated. Then by Lemma 3.4 neither is $\hat{\mathrm{H}}^{*}\left(G, L_{\zeta}\right)$.

Example 3.8. We consider the Klein four group $G=V_{4}$. The classification of the indecomposable $k V_{4}$-modules over a field $k$ of characteristic 2 is well known; see 2, Vol. 1, Thm. 4.3.2] for instance. If the field $k$ is algebraically closed, then every even dimensional indecomposable nonprojective module has the form $L_{\zeta^{m}}$ for some $\zeta \in \mathrm{H}^{1}(G, k)$. On the other hand, every indecomposable module of odd dimension is isomorphic to $\Omega^{i} k$ for some $i$. Because every nonzero element of $\mathrm{H}^{1}(G, k)$ is regular, we have that for any indecomposable $k G$-module $M$, the Tate cohomology of $M$ is finitely generated over $\hat{\mathrm{H}}^{*}(G, k)$ if and only if $V_{G}(M)=V_{G}(k)$. In particular, Conjecture 1.1 holds in this case.

At this point we need to recall a technical notion. We say that a cohomology element $\zeta \in \mathrm{H}^{n}(G, k)$ annihilates the cohomology of a module $M$, if the cup product with $\zeta$ is the zero operator on $\operatorname{Ext}_{k G}^{*}(N, M)$ for all modules $N$. The element $\zeta$ annihilates the cohomology of $M$ if and only if $L_{\zeta} \otimes M \cong \Omega^{n} M \oplus \Omega M \oplus P$ where $P$ is some projective module. See Section 9.7 of 8 . From the same source we have that if $p>2$ and if $\zeta \in \mathrm{H}^{*}(G, k)$ with $n$ even, then $\zeta$ annihilates the cohomology of $L_{\zeta}$.

Even in the case that $p=2$, we know that the degree one elements corresponding to maximal subgroups of a 2-group have the property that $\zeta$ annihilates the cohomology of $L_{\zeta}$. Moreover, the product of any two elements with this property has this property.

We are now prepared to prove the main theorem of this section.

Theorem 3.9. Suppose that $\hat{\mathrm{H}}^{*}(G, k)$ has the property that the product of any two elements in negative degrees is zero. Let $\zeta \in \mathrm{H}^{*}(G, k)$ be a regular element of degree $d$. In the case that $p=2$, assume that $\zeta$ annihilates the cohomology of $L_{\zeta}$. If $M$ is a finitely generated $k G$-module such that $\hat{\mathrm{H}}^{*}(G, M) \neq 0$ and $V_{G}(M) \subseteq V_{G}\langle\zeta\rangle$, then $\hat{\mathrm{H}}^{*}(G, M)$ is not finitely generated as an $\hat{\mathrm{H}}^{*}(G, k)$-module.

Proof. Since $\hat{\mathrm{H}}^{*}(G, M) \neq 0$, by Lemma 3.2 it is enough to show that $\hat{\mathrm{H}}^{*}(G, M)$ has bounded finitely generated submodules. Because of the condition that $V_{G}(M) \subseteq$ $V_{G}\langle\zeta\rangle$, we know that some power of $\zeta$, say $\zeta^{t}$, annihilates the cohomology of $M$. Hence it follows that

$$
L_{\zeta^{t}} \otimes M \cong \Omega M \oplus \Omega^{t d} M \oplus P,
$$

for some projective module $P$. Thus, $\hat{\mathrm{H}}^{*}(G, M)$ has bounded finitely generated submodules if and only if $\hat{\mathrm{H}}^{*}\left(G, L_{\zeta^{t}} \otimes M\right)$ also has this property. Note that if $p>2$, 
then the degree of $\zeta$ must be even because $\zeta$ is regular and hence nonnilpotent. So for any value of $p$ we have that $\zeta$ annihilates the cohomology of $L_{\zeta}$.

The action of $\hat{\mathrm{H}}^{*}(G, k)$ on $\hat{\mathrm{H}}^{*}\left(G, L_{\zeta^{t}} \otimes M\right)$ factors through the map $\hat{\mathrm{H}}^{*}(G, k) \longrightarrow$ $\widehat{\operatorname{Ext}}_{k G}^{*}\left(L_{\zeta^{t}}, L_{\zeta^{t}}\right) \cong \mathrm{H}^{*}\left(G,\left(L_{\zeta^{t}}\right)^{*} \otimes L_{\zeta^{t}}\right) \cong \mathrm{H}^{*}\left(G, \Omega^{-d t} L_{\zeta^{t}} \oplus \Omega^{-1} L_{\zeta^{t}}\right)$, since for any $\zeta$ of degree $d$ we have that $L_{\zeta}^{*} \cong \Omega^{-d-1} L_{\zeta}$ (see [8, Section 11.3). So the target of that map has bounded finitely generated submodules.

Now let $m$ be any integer. Without loss of generality, we can assume that $m<0$. Let

$$
\mathcal{M}=\bigoplus_{n \geq m} \hat{\mathrm{H}}^{n}\left(G, L_{\zeta^{t}} \otimes M\right) \subseteq\left(\bigoplus_{n \geq m} \widehat{\operatorname{Ext}}_{k G}^{n}\left(L_{\zeta^{t}}, L_{\zeta^{t}}\right)\right)\left(\bigoplus_{n \geq m} \hat{\mathrm{H}}^{n}\left(G, L_{\zeta^{t}} \otimes M\right)\right) .
$$

From Definition 3.1, we know that there exists a number $N$ such that

$$
\hat{\mathrm{H}}^{*}(G, k) \cdot \bigoplus_{n \geq m} \widehat{\operatorname{Ext}}_{k G}^{n}\left(L_{\zeta^{t}}, L_{\zeta^{t}}\right) \subseteq \bigoplus_{n \geq N}{\widehat{\operatorname{Ext}_{k G}^{n}}}_{k}^{n}\left(L_{\zeta^{t}}, L_{\zeta^{t}}\right) \text {. }
$$

Hence, we have that

$$
\begin{aligned}
\hat{\mathrm{H}}^{*}(G, k) \cdot \mathcal{M} & \subseteq \hat{\mathrm{H}}^{*}(G, k) \cdot\left(\bigoplus_{n \geq m} \widehat{\operatorname{Ext}}_{k G}^{n}\left(L_{\zeta^{t}}, L_{\zeta^{t}}\right)\right)\left(\bigoplus_{n \geq m} \hat{\mathrm{H}}^{n}\left(G, L_{\zeta^{t}} \otimes M\right)\right) \\
& \subseteq\left(\bigoplus_{n \geq N} \widehat{\operatorname{Ext}}_{k G}^{n}\left(L_{\zeta^{t}}, L_{\zeta^{t}}\right)\right)\left(\bigoplus_{n \geq m} \hat{\mathrm{H}}^{n}\left(G, L_{\zeta^{t}} \otimes M\right)\right) \\
& \subseteq \bigoplus_{n \geq N+m} \hat{\mathrm{H}}^{n}\left(G, L_{\zeta^{t}} \otimes M\right) .
\end{aligned}
$$

Therefore, $\hat{\mathrm{H}}^{*}\left(G, L_{\zeta^{t}} \otimes M\right)$ has bounded finitely generated submodules.

Using the results of the theorem, we can settle Conjecture 1.1 in some special cases as in the following.

Corollary 3.10. Let $p>2$. Suppose that the group $G$ has an abelian Sylow $p$ subgroup with $p$-rank at least two. If $M$ is a finitely generated $k G$-module with $\mathrm{H}^{*}(G, M) \neq 0$ and if $V_{G}(M)$ is a proper subvariety of $V_{G}(k)$, then $\hat{\mathrm{H}}^{*}(G, M)$ is not finitely generated as a module over $\hat{\mathrm{H}}^{*}(G, k)$.

Proof. If $V_{G}(M)$ is a proper subvariety of $V_{G}(k)$, then $V_{G}(M) \subseteq V_{G}(\zeta)$ for some nonnilpotent element $\zeta \in \mathrm{H}^{*}(G, k)$. But because the Sylow subgroup of $G$ is an abelian $p$-group, every nonnilpotent element in $\mathrm{H}^{*}(G, k)$ is regular, and moreover, any two elements in negative degrees in $\hat{\mathrm{H}}^{*}(G, k)$ have zero product. So the proof is complete by the previous theorem.

Remark 3.11. We should note that $\hat{\mathrm{H}}^{*}(G, M)$ having infinitely generated Tate cohomology does not require that it have bounded finitely generated submodules or even submaximal growth of finitely generated submodule (see Remark 3.3). For an example, consider the semidihedral 2-group $G$ of order 16 and let $k=\mathbb{F}_{2}$. Let $M=L_{\zeta}$, where $\zeta \in \mathrm{H}^{1}\left(G, \mathbb{F}_{2}\right)$ is a nonnilpotent element. See the example in Section 4 of [3]. Then it can be seen that $M \cong \Omega k_{H}^{\uparrow G}$ where $H$ is the subgroup defined by the class $\zeta$, that is, the maximal subgroup of $G$ on which $\zeta$ vanishes. So we see that $M \otimes M \cong \Omega M \oplus \Omega M \oplus(k G)^{12}$. Hence, we can see by the results of the next 
section, that $\hat{\mathrm{H}}^{*}(G, M)$ is not finitely generated as a module over $\hat{\mathrm{H}}^{*}(G, k)$. On the other hand, $\zeta$ is not a regular element, so the module $\mathcal{K}^{*}$, which is the kernel of $\zeta$, does not have bounded finitely generated submodules or submaximal growth of finitely generated submodules. However, a careful analysis shows that $\mathcal{K}^{*}$ is not finitely generated.

We end this section by showing that there is a counterexample to the converse of our Conjecture 1.1. We suspect that such examples are numerous. We give only an outline of the proof in one example, leaving the details to the reader.

Proposition 3.12. There exists a module $M$ with $V_{G}(M)=V_{G}(k)$ such that $\hat{\mathrm{H}}^{*}(G, M)$ is not finitely generated over $\hat{\mathrm{H}}^{*}(G, k)$.

Sketch of Proof. Let $G=\langle x, y\rangle$ be an elementary abelian group of order $p^{2}$. Here $k$ has characteristic $p$. We assume that $p>2$. Let $H=\langle y\rangle$ and let $L=k_{H}^{\uparrow G}$ be the induced module. The module of our example is the extension $M$ in the nonsplit exact sequence

$$
\mathcal{E}: \quad 0 \longrightarrow k \stackrel{\sigma}{\longrightarrow} M \longrightarrow L \longrightarrow 0 .
$$

The module $M$ can be described by generators and relations as the quotient of $k G$ by the ideal generated by $(y-1)^{2}$ and $(x-1)(y-1)$. The map $\sigma$ sends 1 to $y-1$. Note that because the dimension of $M$ is relatively prime to $p$, we must have that $V_{G}(M)=V_{G}(k)$.

We have a sequence $0 \rightarrow \mathcal{J}^{*} \rightarrow \hat{\mathrm{H}}^{*}(G, M) \rightarrow \mathcal{K}^{*} \rightarrow 0$ as in 3.4 where $\mathcal{J}^{*}$ and $\mathcal{K}^{*}$ are, respectively, the cokernel and kernel of the map $\hat{\mathrm{H}}^{*}(G, L) \longrightarrow \hat{\mathrm{H}}^{*+1}(G, k)$ given by multiplying by the class of $\mathcal{E}$. Our interest is in the submodule $\mathcal{K}^{*} \subseteq \hat{\mathrm{H}}^{*}(G, L)$. Because $L=k_{H}^{\uparrow G}$, we have by the Eckmann-Shapiro Lemma that $\hat{\mathrm{H}}^{*}(G, L) \cong$ $\hat{\mathrm{H}}^{*}(\langle y\rangle, k)$. Consequently, $\hat{\mathrm{H}}^{*}(G, L)$ has dimension one in every degree and the action of $\hat{\mathrm{H}}^{*}(G, k)$ on $\hat{\mathrm{H}}^{*}(G, L)$ factors through the restriction map $\hat{\mathrm{H}}^{*}(G, k) \longrightarrow$ $\hat{\mathrm{H}}^{*}(\langle y\rangle, k)$, which we know is the zero map in negative degrees. Therefore $\mathcal{K}^{*}$ has bounded finitely generated submodules. So by Lemmas 3.2 and 3.4 , the proof is complete when we show that $\mathcal{K}^{*}$ is not zero in infinitely many negative degrees.

We take the long exact sequence in cohomology corresponding to the dual $\mathcal{E}^{*}$ of the exact sequence $\mathcal{E}$, noting that the module $L$ is self-dual. The connecting homomorphism is a cup product with the class of the sequence $\mathcal{E}^{*}$. By EckmannShapiro, it is the restriction map followed by the cup product with a nonzero class $\eta$ in $\mathrm{H}^{1}(H, k)$. Since $\eta^{2}=0$, we have that the image has dimension one, if $n$ is even and $\delta$ is the zero map if $n$ is odd. Hence because $\operatorname{Dim~}^{n}(G, k)=n+1$, we must also have that $\mathrm{H}^{n}\left(G, M^{*}\right)$ also has dimension $n+1$.

By Tate duality, $\mathrm{H}^{-n}(G, M)$ is dual to $\mathrm{H}^{n-1}\left(G, M^{*}\right)$ for $n>0$. Therefore $\mathrm{H}^{-n}(G, M)$ has dimension $n$, which is the same as the dimension of $\mathrm{H}^{-n}(G, k)$. Returning to the long exact sequence corresponding to $\mathcal{E}$, we argue by dimensions that the connecting homomorphism is the zero map in every second degree. So we show that the dimension of $\mathcal{K}^{n}$ is zero if $n$ is negative and even and is one otherwise. This completes the proof.

\section{Periodic modules}

In this section, we present our second piece of evidence for Conjecture 1.1. We show that for any group $G$ with $p$-rank at least 2 , there is a finitely generated 
module $M$ with the property that $\hat{\mathrm{H}}^{*}\left(G, \operatorname{End}_{k} M\right)$ is not finitely generated as a $\hat{\mathrm{H}}^{*}(G, k)$-module.

We recall that a finite group $G$ has periodic cohomology, meaning that the trivial module $k$ is periodic, if and only if $G$ has $p$-rank zero or one (see [2] or [8]).

Theorem 4.1. Suppose that the group $G$ has p-rank at least 2. Let $M$ be a nonprojective periodic $k G$-module such that $\mathrm{H}^{*}(G, M) \neq 0$. Then $\hat{\mathrm{H}}^{*}\left(G, \operatorname{Hom}_{k}(M, M)\right)$ $\cong \widehat{\operatorname{Ext}}_{k G}^{*}(M, M)$ is not finitely generated as a $\hat{\mathrm{H}}^{*}(G, k)$-module. Thus for any finite group $G$ such that $p$ divides the order of $G$, the Tate cohomology of every finitely generated $k G$-module is finitely generated over $\hat{\mathrm{H}}^{*}(G, k)$ if and only if $G$ has p-rank one, meaning that the Sylow p-subgroup of $G$ is either a cyclic group or a generalized quaternion group.

Proof. Let $E=\left\langle x_{1}, \ldots, x_{n}\right\rangle$ be a maximal elementary abelian $p$-subgroup such that the restriction $M_{E}$ is not a free module. There exists an element $\alpha=\left(\alpha_{1}, \ldots, \alpha_{n}\right) \in$ $k^{n}$ and a corresponding cyclic shifted subgroup $\left\langle u_{\alpha}\right\rangle$,

$$
u_{\alpha}=1+\sum_{i=1}^{n} \alpha_{i}\left(x_{i}-1\right)
$$

such that the restriction of $M$ to $\left\langle u_{\alpha}\right\rangle$ is not projective (see Section 5.8 of [2]). Hence, the identity homomorphism $\operatorname{Id}_{M}: M \longrightarrow M$ does not factor through a projective $k\left\langle u_{\alpha}\right\rangle$-module. As a consequence, the map $k \longrightarrow \operatorname{Hom}_{k}(M, M)$ which sends $1 \in k$ to $\operatorname{Id}_{M}$ must represent a nonzero class in $\hat{\mathrm{H}}^{0}\left(\left\langle u_{\alpha}\right\rangle, \operatorname{Hom}_{k}(M, M)\right)$.

The next thing that we note is that the restriction map

$$
\operatorname{res}_{G,\left\langle u_{\alpha}\right\rangle}: \hat{\mathrm{H}}^{d}(G, k) \longrightarrow \hat{\mathrm{H}}^{d}\left(\left\langle u_{\alpha}\right\rangle, k\right)
$$

is the zero map if $d<0$. The reason is that the restriction map

$$
\operatorname{res}_{E,\left\langle u_{\alpha}\right\rangle}: \hat{\mathrm{H}}^{d}(E, k) \longrightarrow \hat{\mathrm{H}}^{d}\left(\left\langle u_{\alpha}\right\rangle, k\right)
$$

is zero by [3] since $E$ has rank at least 2 .

Now suppose that $M$ is periodic of period $t$. For every $m$ we have that $\Omega^{m t} M \cong$ $M$ and there exists an element

$$
\zeta_{m} \in \widehat{\operatorname{Ext}}_{k G}^{m t}(M, M) \cong \hat{\mathrm{H}}^{m t}\left(G, \operatorname{Hom}_{k}(M, M)\right)
$$

such that $\zeta_{m}$ is not zero on restriction to $\left\langle u_{\alpha}\right\rangle$. That is, $\zeta_{m}$ is represented by a cocycle

$$
k \quad \longrightarrow \quad \operatorname{Hom}_{k}(M, M) \cong \Omega^{m t} \operatorname{Hom}_{k}(M, M)
$$

which does not factor through a projective module on restriction to $\left\langle u_{\alpha}\right\rangle$.

Suppose that $\hat{\mathrm{H}}^{*}\left(G, \operatorname{Hom}_{k}(M, M)\right)$ is finitely generated as a module over $\hat{\mathrm{H}}^{*}(G, k)$. Then there exist generators $\mu_{1}, \ldots, \mu_{r}$ of $\hat{\mathrm{H}}^{*}\left(G, \operatorname{Hom}_{k}(M, M)\right)$, having degrees $d_{1}, \ldots, d_{r}$, respectively. Choose an integer $m$ such that $m t<\min \left\{d_{i}\right\}$. We must have that $\zeta_{m}=\sum_{i=1}^{r} \gamma_{i} \mu_{i}$ for some $\gamma_{i} \in \hat{\mathrm{H}}^{m t-d_{i}}(G, k)$. But now, for every $i$, we have that $m t-d_{i}$ is negative. Hence $\operatorname{res}_{G,\left\langle u_{\alpha}\right\rangle}\left(\gamma_{i}\right)=0$ for every $i$. Therefore, since restriction onto a shifted subgroup is a homomorphism we have that $\operatorname{res}_{G,\left\langle u_{\alpha}\right\rangle}\left(\zeta_{m}\right)=0$; but this is a contradiction.

To prove the last statement of the theorem, we recall that every finite group with nontrivial Sylow $p$-subgroup admits a finitely generated nonprojective and periodic $k G$-module in the thick subcategory generated by $k$. If the group has $p$-rank one, 
then $k$ is such a module. If the $p$-rank of $G$ is greater than one, then some tensor product $L_{\zeta_{1}} \otimes \cdots \otimes L_{\zeta_{n}}$ is periodic and is in the thick subcategory generated by $k$, provided the dimension of the variety $V_{G}\left(\zeta_{1}\right) \cap \cdots \cap V_{G}\left(L_{\zeta_{n}}\right)$ has dimension one (see Chapter 10 of [8] or Chapter 5 of [2], Volume 2).

There is one other concept which ties up well with finite generation of Tate cohomology, and this is a ghost projective class in the $\operatorname{stmod}(k G)$. Consider the pair $(\mathcal{P}, \mathcal{G})$, where $\mathcal{P}$ is a class of objects isomorphic in $\operatorname{stmod}(k G)$ to finite direct sums of suspensions of $k$, and $\mathcal{G}$ is a class of all ghosts in $\operatorname{stmod}(k G)$. Recall that a ghost is a map of $k G$-modules that is zero in Tate cohomology. We say that $(\mathcal{P}, \mathcal{G})$ is a ghost projective class if the following 3 conditions are satisfied.

(1) The class of all maps $X \rightarrow Y$ such that the composite $P \rightarrow X \rightarrow Y$ is zero for all $P$ in $\mathcal{P}$ and all maps $P \rightarrow X$ is precisely $\mathcal{G}$.

(2) The class of all objects $P$ such that the composite $P \rightarrow X \rightarrow Y$ is zero for all maps $X \rightarrow Y$ in $\mathcal{G}$ and all maps $P \rightarrow X$ is precisely $\mathcal{P}$.

(3) For each object $X$ there is an exact triangle $P \rightarrow X \rightarrow Y$ with $P$ in $\mathcal{P}$ and $X \rightarrow Y$ in $\mathcal{G}$.

The first question that comes to mind is whether the ghost projective class exists in $\operatorname{stmod}(k G)$. We answer this in the next theorem.

Theorem 4.2. For $G$ a finite group, such that $p$ divides the order of $G$. The ghost projective class exists in $\operatorname{stmod}(k G)$ if and only if $G$ has p-rank one.

Proof. It is clear from the definition of a ghost that $\mathcal{P}$ and $\mathcal{G}$ are orthogonal, i.e., the composite $P \rightarrow M \stackrel{h}{\rightarrow} N$ is zero for all $P$ in $\mathcal{P}$, for all $h$ in $\mathcal{G}$, and all maps $P \rightarrow M$. So by [12, Lemma 3.2] it remains to show that for all finitely generated $k G$-modules $M$, there exists a triangle $P \rightarrow M \rightarrow N$ such that $P$ is in $\mathcal{P}$ and $M \rightarrow N$ is in $\mathcal{G}$. The exact triangle (2.1) has this form in the case that the Tate cohomology of $M$ is finitely generated over $\hat{\mathrm{H}}^{*}(G, k)$.

For the converse, suppose that $M$ is a finitely generated $k G$-module. Since the ghost projective class exists, we have an exact triangle

$$
\bigoplus \Omega^{i} k \stackrel{\oplus \theta_{i}}{\longrightarrow} M \stackrel{\rho}{\longrightarrow} N
$$

in $\operatorname{stmod}(k G)$ where $\rho$ is a ghost. We claim that the finite set $\left\{\theta_{i}\right\}$ generate $\hat{\mathrm{H}}^{*}(G, M)$ as a module over $\hat{\mathrm{H}}^{*}(G, k)$. To see this, consider any element $\gamma$ in $\hat{\mathrm{H}}^{t}(G, M)$ represented by a cocycle $\gamma: \Omega^{t} k \rightarrow M$. Since $\rho$ is a ghost, we get the following commutative diagram:

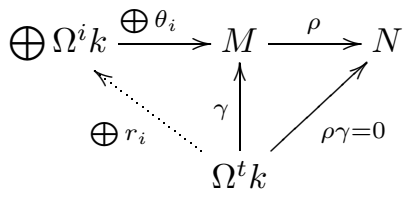

From this diagram, we infer that $\gamma=\sum r_{i} \theta_{i}$. This shows that $\hat{\mathrm{H}}^{*}(G, M)$ is finitely generated, as desired. Hence, by Theorem 4.1 the $p$-rank of $G$ is one.

\section{Modules With finitely Generated Tate COHOMOlogy}

It is clear that any module $M$ which is a direct sum of Heller translates $\Omega^{n} k$ has finitely generated Tate cohomology. This is simply because $\hat{\mathrm{H}}^{*}(G, M)$ is a direct 
sum of copies of $\hat{\mathrm{H}}^{*}(G, k)$ which have been suitably translated in degrees. Also, any finitely generated modules over a group with periodic cohomology has finitely generated Tate cohomology. In this section we show that in general there are many more modules with this property. Notice that every one of the modules which we discuss has the property that $V_{G}(M)=V_{G}(k)$, consistent with Conjecture 1.1.

We first consider the Tate cohomology of modules $M$ which can occur as the middle term of an exact sequence of the form

$$
0 \longrightarrow \Omega^{m} k \longrightarrow M \longrightarrow \Omega^{n} k \longrightarrow 0
$$

for some values of $m$ and $n$. Such a sequence represents an element $\zeta$ in

$$
\operatorname{Ext}_{k G}^{1}\left(\Omega^{n} k, \Omega^{m} k\right) \cong \widehat{\operatorname{Ext}}_{k G}^{n+1-m}(k, k) \cong \hat{\mathrm{H}}^{n+1-m}(G, k) .
$$

For the purposes of examining the Tate cohomology of $M$ there is no loss of generality in applying the shift operator $\Omega^{-m}$. Consequently, we can assume that the sequence has the form

$$
0 \longrightarrow k \longrightarrow M \longrightarrow \Omega^{n} k \longrightarrow 0
$$

for some $n$ and that $\zeta \in \hat{\mathrm{H}}^{n+1}(G, k)$.

The principal result of this section is the following.

Theorem 5.1. Suppose that the cohomology of $G$ is not periodic and that for the module $M$ and cohomology element $\zeta$ as above, the map

$$
\zeta: \hat{\mathrm{H}}^{*}(G, k) \longrightarrow \hat{\mathrm{H}}^{*}(G, k)
$$

given by multiplication by $\zeta$ has a finite dimensional image. Then the Tate cohomology $\hat{\mathrm{H}}^{*}(G, M)$ is finitely generated as a module over $\hat{\mathrm{H}}^{*}(G, k)$.

There are many examples of sequences satisfying the conditions of the theorem. In particular, it is often the case that multiplication by an element $\zeta$ in negative cohomology will have finite dimensional image. An example is the element in degree -1 which represents the almost split sequence for the module $k$. Details of this example are given below. In addition, if the depth of $\mathrm{H}^{*}(G, k)$ is two or more, then all products involving elements in negative degrees are zero, and the principal ideal generated by any element in negative cohomology contains no nonzero elements in positive degrees (see [3]). Hence, multiplication by any element $\zeta$ in negative cohomology has finite dimensional image.

Proof. As in Lemma 3.4, we have an exact sequence of $\hat{\mathrm{H}}^{*}(G, k)$-modules

$$
0 \longrightarrow \mathcal{J}^{*} \longrightarrow \hat{\mathrm{H}}^{*}(G, M) \longrightarrow \mathcal{K}^{*}[-n] \longrightarrow 0
$$

where $\mathcal{K}^{*}$ is the kernel of multiplication by $\zeta$ on $\hat{\mathrm{H}}^{*}(G, k)$ and $\mathcal{J}^{*}$ is the cokernel. By assumption, the image of multiplication by $\zeta$ has finite total dimension. This means that in all but a finite number of degrees $r$, multiplication by $\zeta$ is the zero map. Clearly, $\mathcal{J}^{*}$ is finitely generated over $\hat{\mathrm{H}}^{*}(G, k)$. So, $\hat{\mathrm{H}}^{*}(G, M)$ is finitely generated as a module over $\hat{\mathrm{H}}(G, k)$ if and only if $\mathcal{K}^{*}$ has the same property.

First we view $\mathcal{K}^{*}$ as a module over the ordinary cohomology $\operatorname{ring} \mathrm{H}^{*}(G, k)$. The elements in nonnegative degrees form a submodule $\mathcal{M}^{*}=\sum_{i \geq 0} \mathcal{K}^{i}$, which is finitely generated over $\mathrm{H}^{*}(G, k)$. Let $\mathcal{N}^{*}$ be the $\hat{\mathrm{H}}^{*}(G, k)$-submodule module of $\mathcal{K}^{*}$ generated by $\mathcal{M}^{*}$. Our objective is to show that $\mathcal{N}^{*}=\mathcal{K}^{*}$, thereby proving the 
finite generation of $\mathcal{K}^{*}$. We notice first that $\mathcal{K}^{n} \subseteq \mathcal{N}^{*}$ for $n \geq 0$. It remains only to show the same for $n<0$.

Because the quotient of $\hat{\mathrm{H}}^{*}(G, k)$ by $\mathcal{K}^{*}$ is finite dimensional, we must have that $\hat{\mathrm{H}}^{n}(G, k)=\mathcal{K}^{n}$ for $n$ sufficiently large. For some sufficiently large $n$, we can find an element $\gamma$ in $\mathcal{K}^{n}$ which is a regular element for the ordinary cohomology ring $\mathrm{H}^{*}(G, k)$. For example, by Duflot's Theorem (see Theorem 3.6), any element whose restriction to the center of a Sylow $p$-subgroup of $G$ is not nilpotent will serve this purpose (see [2] or [8]). Let $\theta$ be the image of $\gamma$ in $\mathcal{K}^{n}$. We know that $\theta$ is not zero. We also know that multiplication by $\gamma$ is a surjective map

$$
\gamma: \hat{\mathrm{H}}^{m-n}(G, k) \longrightarrow \hat{\mathrm{H}}^{m}(G, k)
$$

whenever $m<0$ (see Lemma 3.5 of [3]). Hence, for any $m<0$, we must have that $\hat{\mathrm{H}}^{m-n}(G, k) \theta=\mathcal{K}^{m}$. Since $\theta \in \mathcal{N}^{*}$, we get that $\mathcal{K}^{m} \subseteq \mathcal{N}^{*}$ for all $m<0$. Hence, $\mathcal{K}^{*}=\mathcal{N}^{*}$ is finitely generated as a module over $\hat{\mathrm{H}}^{*}(G, k)$.

One application of the theorem is the following.

Corollary 5.2. The middle term of the almost split sequence

$$
0 \longrightarrow \Omega^{2} k \stackrel{\sigma}{\longrightarrow} M \longrightarrow k \longrightarrow 0
$$

ending with $k$ has finitely generated Tate cohomology.

Proof. If $G$ has $p$-rank zero or one, then by Theorem 4.1, all modules have finitely generated Tate cohomology. So we assume that $G$ has $p$-rank at least 2 . The almost split sequence corresponds to an element $\zeta$ in $\hat{\mathrm{H}}^{-1}(G, k)$. One of the defining properties of the almost split sequence is that for any module $N$, the connecting homomorphism $\delta$ in the corresponding sequence

$$
\ldots \longrightarrow \operatorname{Hom}_{k G}(N, M) \stackrel{\sigma^{*}}{\longrightarrow} \operatorname{Hom}_{k G}(N, k) \stackrel{\delta}{\longrightarrow} \operatorname{Ext}_{k G}^{1}\left(N, \Omega^{2} k\right) \longrightarrow \ldots
$$

is nonzero if and only if $N \cong k$. This connecting homomorphism is multiplication by $\zeta$. Now any element $\gamma$ in $\hat{\mathrm{H}}^{d}(G, k)$ is represented by a map $\gamma: \Omega^{-d} k \rightarrow k$. Hence, we see that $\zeta \gamma=0$ whenever $d \neq 0$. Therefore, multiplication by $\zeta$ on $\hat{\mathrm{H}}^{*}(G, k)$ has finite dimensional image.

Proposition 5.3. Let $N$ be a finitely generated indecomposable nonprojective $k G$ module that is not isomorphic to $\Omega^{i} k$ for any $i$. Consider the almost split sequence

$$
0 \longrightarrow \Omega^{2} N \longrightarrow M \longrightarrow N \longrightarrow 0
$$

ending in $N$. If $N$ has finitely generated Tate cohomology, then so does the middle term $M$.

Proof. In a similar way as in the previous proof, for any $i$, the connecting homomorphism $\delta$ in the sequence

$$
\ldots \longrightarrow \operatorname{Hom}_{k G}\left(\Omega^{i} k, M\right) \longrightarrow \operatorname{Hom}_{k G}\left(\Omega^{i} k, N\right) \stackrel{\delta}{\longrightarrow} \operatorname{Ext}_{k G}^{1}\left(\Omega^{i} k, \Omega^{2} N\right) \longrightarrow \ldots
$$

is zero because $\Omega^{i} k \neq N$. As a consequence, $\delta$ induces the zero map on Tate cohomology. Hence, the long exact sequence in Tate cohomology breaks into short exact sequences:

$$
0 \longrightarrow \hat{\mathrm{H}}^{*}\left(G, \Omega^{2} N\right) \longrightarrow \hat{\mathrm{H}}^{*}(G, M) \longrightarrow \hat{\mathrm{H}}^{*}(G, N) \longrightarrow 0 .
$$


It is now clear that if $N$ has finitely generated Tate cohomology, then so does $M$.

In summary, combining the last two results we have the following theorem.

Theorem 5.4. Let $C$ be a connected component of the stable Auslander-Reiten quiver associated to $k G$. Then either all modules in $C$ have finitely generated Tate cohomology or no module in $C$ has this property. Moreover, all modules in the connected component of the quiver which contains $k$ have finitely generated Tate cohomology.

It is shown in 1, Proposition 5.2] that all modules $M$ in the connected component of the quiver which contains $k$ have the property $V_{G}(M)=V_{G}(k)$. Thus the last theorem is consistent with Conjecture 1.1.

\section{ACKNOWLEDGMENTS}

The first author is grateful to the RWTH in Aachen for their hospitality and the Alexander von Humboldt Foundation for financial support during a visit to Aachen during which parts of this paper were written. The first and second authors had some fruitful conversations on this work at MSRI during the spring 2008 semester on Representation Theory and related topics. They both would like to thank MSRI for its hospitality.

\section{REFERENCES}

[1] M. Auslander and J. F. Carlson, Almost-split sequences and group rings. J. Algebra, 103(1):122-140, 1986. MR860693 (88a:16054)

[2] D. J. Benson, Representations and Cohomology, I, II, Cambridge Univ. Press, Cambridge, 1991. MR.1110581 (92m:20005)

[3] D. J. Benson and J. F. Carlson, Products in negative cohomology, J. Pure Appl. Algebra, 82(1992), 107-129. MR.1182934 (93i:20058)

[4] D. J. Benson, J. F. Carlson and J. Rickard, Thick subcategories of the stable category, Fund. Math. 153(1997), 59-80. MR.1450996 (98g:20021)

[5] D. J. Benson, J. F. Carlson, and G. R. Robinson, On the vanishing of group cohomology, J. Algebra, 131(1):40-73, 1990. MR1054998 (91c:20073)

[6] W. Burnside, Theory of groups of finite order, Dover Publications Inc., New York, 1955. 2nd ed. MR0069818 (16:1086c)

[7] J. F. Carlson, Modules and group algebras. Lectures in Mathematics ETH Zürich. Birkhäuser Verlag, Basel, 1996. Notes by Ruedi Suter. MR.1393196 (97c:20013)

[8] J. Carlson, L. Townsley, L. Valero-Elizondo and M. Zhang, Cohomology Rings of Finite Groups, Kluwer, Dordrecht, 2003. MR2028960 (2004k:20110)

[9] H. Cartan and S. Eilenberg, Homological algebra. Princeton Landmarks in Mathematics. Princeton University Press, Princeton, NJ, 1999. With an appendix by David A. Buchsbaum, reprint of the 1956 original. MR 1731415 (2000h:18022)

[10] S. K. Chebolu, J. D. Christensen, and J. Mináč, Groups which do not admit ghosts. Proc. Amer. Math. Soc., 136:1171-1179, 2008. MR2367091 (2008k:20017)

[11] S. K. Chebolu, J. D. Christensen, and J. Mináč, Ghosts in modular representation theory, Advances in Mathematics, 217:2782-2799, 2008. MR.2397466 (2008m:20018)

[12] J. D. Christensen, Ideals in triangulated categories: phantoms, ghosts and skeleta, Adv. Math., 136(2):284-339, 1998. MR1626856 (99g:18007)

[13] R. G. Swan, Groups with periodic cohomology. Bull. Amer. Math. Soc., 65:368-370, 1959. MR0115175 (22:5977)

[14] J. Tate, The higher dimensional cohomology groups of class field theory. Ann. of Math. (1), 56:294-297, 1952. MR0049950(14:252b) 
Department of Mathematics, University of Georgia, Athens, Georgia 30602

E-mail address: jfc@math.uga.edu

Department of Mathematics, Illinois State University, Campus box 4520, Normal, ILLINOIS 61790

E-mail address: schebol@ilstu.edu

Department of Mathematics, University of Western Ontario, London, On N6A 5B7, CANADA

E-mail address: minac@uwo.ca 